\title{
Incidence and electrophysiological characteristics of spontaneous ventricular tachyarrhythmias in high risk coronary patients and prophylactic implantation of a defibrillator
}

\author{
C Wolpert, J Kuschyk, N Aramin, S Spehl, F Streitner, T Süselbeck, B Schumacher, K K Haase, \\ R Schimpf, M Borggrefe
}

Heart 2004;90:667-671. doi: 10.1136/hrt.2003.019042

See end of article for authors' affiliations

Correspondence to: Dr C Wolpert, 1st Department of MedicineCardiology, University Hospital Mannheim, Theodor-Kutzer-Ufer 1-3, 68167 Mannheim, Germany; christian. wolpert@med.ma.uniheidelberg.de

Accepted 25 September 2003

\begin{abstract}
Objectives: To assess the incidence and electrophysiological characteristics of spontaneous ventricular tachyarrhythmias after implantable cardioverter-defibrillator (ICD) implantation for primary prevention. Design: Prospective observational study.

Patients: 41 consecutive patients, who fulfilled MADIT (multicenter automatic defibrillator implantation trial) I criteria, except for suppressibility by procainamide, and who received a prophylactic ICD.

Interventions: Subpectoral implantation of an ICD.

Main outcome measures: Incidence of ventricular tachyarrhythmias and their electrophysiological characteristics with respect to timing of the arrhythmia, tachyarrhythmia cycle length, mode of termination, and clinical relevance.

Results: During a mean (SD) follow up of 30 (21) months 18 of 41 (43.9\%) patients experienced 142 appropriate ICD treatments. The mean (SD) time to first event was 9.6 (15.1) months. One patient had ventricular fibrillation (VF), 12 patients ventricular tachycardia (VT), and five both VT and VF. The mean (SD) cycle length of monomorphic VT was 306 (42) ms. Of 142 episodes, 117 (82.3\%) were terminated by antitachycardia pacing and another $25(17.6 \%)$ by ICD discharges. Cumulative survival of hypothetical death, defined as treated VT with a cycle length $<260 \mathrm{~ms}$ or VF, was $83.2 \%$ after one year and $78.4 \%$ after two years.

Conclusions: Patients with a left ventricular ejection fraction $<35 \%$, a history of myocardial infarction, non-sustained VT, and inducible VT/VF are at high risk of VT/VF early after implantation. Therefore, implantation of a tiered treatment defibrillator seems to be justified.
\end{abstract}

T he results of the MADIT I (multicenter automatic defibrillator implantation trial) have led to an extension of the indication for implantable cardioverter-defibrillator (ICD) implantation. ${ }^{1}$ However, various modifications of the MADIT I protocol have been introduced because intravenous procainamide is not available in most European countries. Furthermore, serial drug testing to assess suppressibility of ventricular tachyarrhythmias is no longer used. Therefore, especially in Europe the main inclusion criteria for prophylactic ICD implantation are depressed left ventricular function, prior myocardial infarction, documented non-sustained ventricular tachycardia (VT), and inducible ventricular tachyarrhythmias. In postmyocardial infarction patients, there is still a lack of information and for the overall patient population at risk the results of MADIT I are not being fully implemented into everyday decision making. The goal of the present prospective study was to assess the actual incidence and electrophysiological characteristics of spontaneous ventricular tachyarrhythmias after ICD implantation for primary prevention of sudden death in patients with a history of myocardial infarction, left ventricular ejection fraction $<35 \%$, non-sustained VT, and inducible sustained ventricular tachyarrhythmias during programmed ventricular stimulation. Furthermore, the hypothetical clinical relevance was determined by assessing the incidence of potentially life threatening events with a cycle length $<260 \mathrm{~ms}$.

\section{PATIENTS AND METHODS}

During the study period 145 patients who fulfilled the MADIT inclusion criteria for programmed stimulation underwent programmed stimulation to test for inducible ventricular tachyarrhythmias. Forty one of 145 (28.2\%) consecutive patients fulfilling all of the following criteria after induction of VT/ventricular fibrillation (VF) at programmed stimulation were then prospectively enrolled into the study:

- depressed left ventricular function $(<35 \%)$ assessed by left ventriculography

- prior history of myocardial infarction (>1 month)

- documented non-sustained VT $\geqslant 3$ consecutive beats $(\geqslant 120$ beats/min) during Holter monitoring

- inducible sustained monomorphic VT or VF at electrophysiological study (defined as a duration $\geqslant 30$ seconds or requiring cardioversion because of haemodynamic compromise).

Abbreviations: AVID, antiarrhythmics versus implantable defibrillators; CASH, cardiac arrest study Hamburg; CIDS, Canadian implantable defibrillator study; ICD, implantable cardioverter-defibrillator; MADIT, multicenter automatic defibrillator implantation trial; MUST, multicenter unsustained tachycardia trial; NYHA, New York Heart Association; VF, ventricular fibrillation; VT, ventricular tachycardia 
All patients underwent coronary angiography, echocardiography, and Holter ECG monitoring before implantation. Programmed stimulation was performed at both the right ventricular apex and the right ventricular outflow tract with up to three premature stimuli at three driving cycle lengths $(600,500$, and $400 \mathrm{~ms})$ with a $10 \mathrm{~ms}$ decrement of the last coupled extrastimulus and no interpulse cycle length less than 190 ms. Reinducibility was not assessed. In contrast to the original MADIT protocol polymorphic VT or VF was accepted, even if induced by three premature stimuli. When qualifying for ICD implantation, each patient gave written informed consent before ICD implantation. All patients underwent non-thoracotomy placement of the device. Twenty one patients received a single and another 20 patients a dual chamber defibrillator. The device was placed in the left subpectoral region in all patients except for one. Devices were uniformly programmed to a two zone detection based on the information of the inducible VT or by programming empirical detection rates between $130-180$ beats/min for the lowest VT detection boundary. Devices in all patients were programmed to primary antitachycardia pacing attempts followed by shock.

All devices provide extensive data log information and stored endocardial electrograms for further rhythm analysis. Stored endocardial electrograms were analysed by three independent observers to classify the arrhythmia leading to ICD treatment and to avoid inclusion of inappropriate treatments. Classification was based on the following:

- sudden onset of the arrhythmia

- rate stability

- ventriculoatrial association

- intracardiac morphology (polymorphic or monomorphic)

- rate (fast VT defined as cycle length $<260 \mathrm{~ms}$ )

- treatment success.

Patients were seen one month after ICD implantation and thereafter every three months routinely in the outpatient clinic. At each follow up visit all device parameters and thresholds were tested, the device data log was interrogated, and stored electrograms were reviewed.

Table 1 outlines patient demographics at the time of implantation. There were 38 men and three women. The mean (SD) age was 67.4 (8) years. The mean (SD) left ventricular ejection fraction was $27.2(8) \%$. Nineteen patients

\begin{tabular}{lc} 
Table 1 Clinical characteristics of the patients $(\mathrm{n}=41)$ \\
\hline Age (years) & $67.4(8)$ \\
Male sex & $92 \%$ \\
NYHA functional class & $20 \%$ \\
I & $51 \%$ \\
III & $29 \%$ \\
Left ventricular ejection fraction (\%) & $27.2(7.6)$ \\
Myocardial infarction location & \\
Anterior wall & $46 \%$ \\
Inferior wall & $39 \%$ \\
Anterior and inferior wall & $15 \%$ \\
>6 month lag between MI and ICD implantation & $88 \%$ \\
Atrial fibrillation & $29 \%$ \\
Coronary bypass surgery & $54 \%$ \\
Coronary angioplasty & $30 \%$ \\
Medication at last contact & \\
Diuretics & $73 \%$ \\
ACE inhibitor & $83 \%$ \\
$\beta$ Blockers & $69 \%$ \\
Digitalis & $75 \%$ \\
\hline
\end{tabular}

Data are mean $(S D)$ or percentages.

$A C E$, angiotensin converting enzyme; MI, myocardial infarction; NYHA, New York Heart Association. had a history of anterior myocardial infarction, 16 had a history of an inferior infarction, and six patients had a history of both inferior and anterior infarction. The mean (SD) time between the last myocardial infarction and device implantation was 101 (87.2) months (median 84 months). A left ventricular aneurysm was present in 21 patients and $54 \%$ of the patients had previously undergone coronary bypass grafting. Coronary angioplasty had been performed in $30 \%$ of all patients. Sixteen of 41 (39\%) patients had a wide QRS complex defined as $\geqslant 120 \mathrm{~ms}$. Six patients were permanently paced in the ventricle. Twenty per cent of the patients were in New York Heart Association (NYHA) functional class I, 51\% in class II, and the remaining $29 \%$ in class III. Fifty nine per cent of the patients were taking $\beta$ blocking agents at the time of implantation and $69 \%$ at the last follow up. Nine of 41 patients received specific antiarrhythmic agents such as sotalol and amiodarone for concomitant atrial fibrillation or atrial flutter, $83 \%$ of the patients were treated with angiotensin converting enzyme inhibitors, and 73\% were treated with diuretics.

During programmed ventricular stimulation 39 patients had inducible VT and another two patients had inducible VF.

\section{RESULTS}

\section{Incidence of ventricular tachyarrhythmias}

All 41 patients were followed up over a mean (SD) of 30.2 (21) months after ICD implantation. No patient was lost to follow up. Two patients died during the observation period of non-cardiac causes, one of cancer and one of complicated stroke. Eighteen of the 41 patients $(43.9 \%)$ received at least one appropriate ICD treatment during follow up: one patient experienced an episode of VF, another five patients had both monomorphic VT and fibrillation, and 12 patients presented with monomorphic VT (an example is shown in fig 1). Thus, 17 of $18(94 \%)$ patients had monomorphic VT. Among all patients with any ventricular tachyarrhythmia six patients had one episode, nine patients had two to five episodes, and three patients had more than five episodes during follow up.

A comparison between those patients with a non-paced QRS width $\geqslant 120 \mathrm{~ms}$ and those with $<120 \mathrm{~ms}$ showed that patients with a wide QRS complex were more likely to be appropriately treated. Eleven of $16(68.7 \%)$ patients with a wide QRS complex experienced a total of 127 VT/VF episodes during follow up. Seven of $19(36.8 \%)$ patients with a QRS complex $<120 \mathrm{~ms}$ had 10 episodes during follow up. One of six patients with permanent ventricular pacing had five ventricular tachyarrhythmias.

\section{Time to first event}

The mean (SD) time to the first event of a ventricular tachyarrhythmia was 9.6 (15.1) months (median 2.5 months) after ICD implantation. Thirteen of 18 (72.2\%) patients experienced the first event in the first 12 months. In eight of $18(44.4 \%)$ patients the first event occurred during the first four weeks after implantation. Figure 2 shows the Kaplan-Meier curve for arrhythmia-free survival. The actuarial rate for freedom from any VT/VF was $68.5 \%$ and $58.5 \%$ after 12 months and 24 months, respectively. The actuarial rate for freedom from fast VT/VF rate was $83.2 \%$ and $78.4 \%$ at one and two years, respectively. The mean (SD) time from first documented VT/VF to last infarction was 155 (95) months in this patient population.

\section{Characteristics of spontaneous ventricular tachyarrhythmic events}

Of 142 spontaneous ventricular tachyarrhythmias, 131 (92.3\%) were monomorphic VTs. Eight of 142 (5.6\%) episodes were classified as VF and three episodes as polymorphic VT $(2.1 \%)$. The mean (SD) cycle length of the 


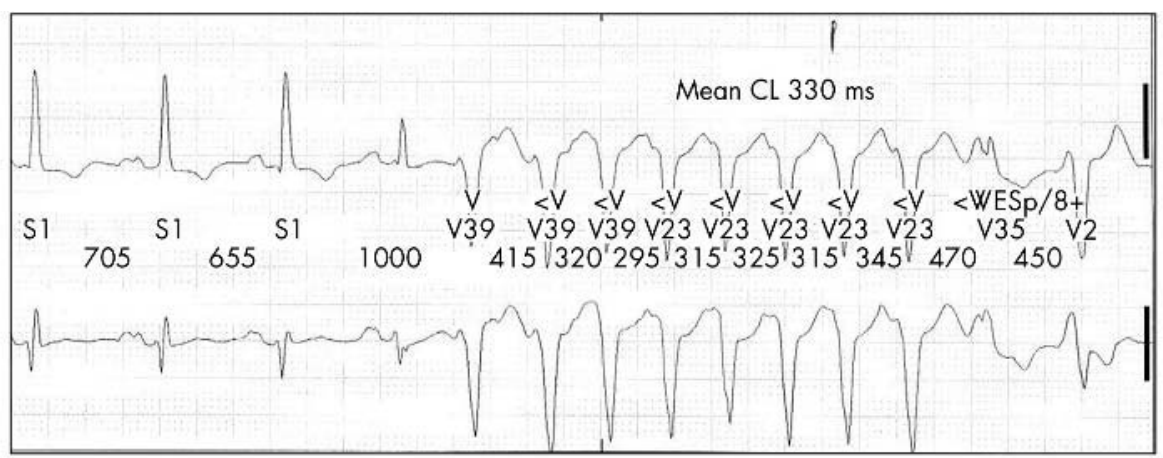

Figure 1 From the same patient (top) a two lead Holter registration of a nonsustained ventricular tachycardia (VT) and (bottom) a stored electrogram strip from the device data log with VT terminated by ventricular antitachycardia burst pacing. $\mathrm{CL}$, cycle length.

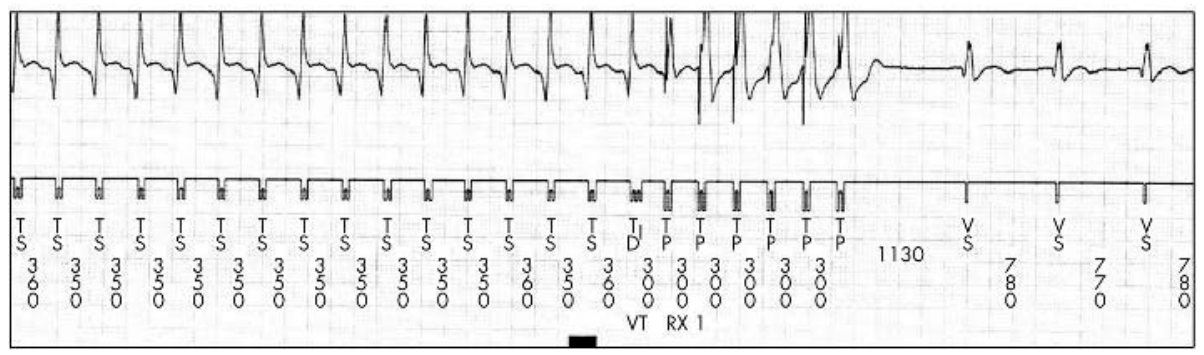

documented VT was 306 (42) ms. No VTs had a cycle length of $<430 \mathrm{~ms}$. Twenty four episodes had a cycle length between 430-320 ms. One hundred and seven episodes had a cycle length $<320 \mathrm{~ms}$. Altogether, 55.6\% (10/18) of the patients with a VT or VF event experienced fast VT (cycle length $<260 \mathrm{~ms}$ ). The minimum time to treatment was 16 cycles or 2.5 seconds for all patients depending on the implanted device and on the manufacturer's detection criterion for initial detection of VT. Antitachycardia pacing successfully treated 117 of $142(82.3 \%)$ episodes. Defibrillator shock terminated 25 (17.6\%) episodes.

The mean (SD) cycle length of induced VT was 264 (53) ms at programmed stimulation. Comparing the VT cycle length of spontaneous VT versus induced VT only two patients had a difference of $<30 \mathrm{~ms}$ between spontaneous and induced VT.

\section{DISCUSSION}

The role of ICDs in secondary prevention is now well established after the AVID (antiarrhythmics versus

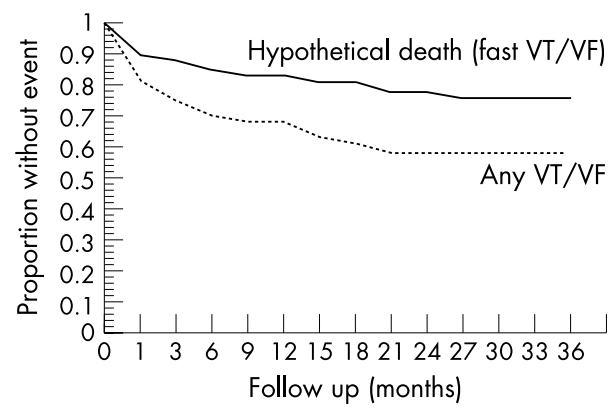

Figure 2 Actuarial survival rates for freedom from any ventricular tachyarrhythmia (dotted line) and freedom from any ventricular fibrillation (VF)/fast VT (<260 ms cycle length) (continuous line) Kaplan-Meier curves for the overall patient population. The actuarial survival rate for freedom from any VT/VF was $68.5 \%$ and $58.5 \%$ after 12 months and 24 months, respectively. The actuarial survival rate for freedom from fast VT/VF was $83.2 \%$ and $78.4 \%$ at one and two years' follow up, respectively. implantable defibrillators) trial, CIDS (Canadian implantable defibrillator study), and CASH (cardiac arrest study Hamburg). ${ }^{2-5}$ The usefulness of ICD treatment in the absence of documented VT/VF (primary prevention) is still under debate. The results of MADIT I have led to an extension of indications, introducing the ICD as a prophylactic treatment for patients with coronary artery disease, severely depressed left ventricular function, prior myocardial infarction, documented non-sustained VT during Holter monitoring, and inducible VT/VF. ${ }^{1}$ However, in MADIT I non-suppressibility of ventricular tachyarrhythmias after intravenous procainamide has been a precondition for randomisation. In MADIT II, inducibility and suppressibility of VT/VF were no longer necessary inclusion criteria. Patients with severely depressed left ventricular function were shown to have a lower mortality when treated with ICDs. ${ }^{6}$ Although the results of MADIT I and MADIT II have not yet been confirmed by other studies, both indications have been incorporated into the guidelines for ICD implantation of the American Heart Association, which have been updated recently. ${ }^{7}$ In Germany, for example, the MADIT I criteria have been adopted by the guidelines in a modified way, but the MADIT II criteria have not been addressed by the German Cardiological Society and are defined only as a class IIa indication by the European Society of Cardiology. ${ }^{8} 9$ The main reason for this is the lack of confirming trials and clinical reports dealing with the actuarial risk of ventricular tachyarrhythmias in these selected patients.

There are four major findings in the present study. Firstly, there was a high incidence of spontaneous ventricular tachyarrhythmias; secondly, more than $90 \%$ of all episodes recorded during follow up were monomorphic VT; thirdly, there was a high incidence of fast VT (VT cycle length $<260 \mathrm{~ms}$ ); and lastly, there was a long time interval between MI and the first VT/VF episode.

\section{Incidence of VT/VF}

In the present study the overall incidence of ventricular tachyarrhythmias after ICD implantation was $43.9 \%$, which is close to that observed in patients who survived a sudden death event or presented with haemodynamically untolerated 
VTs ( secondary prevention). ${ }^{1}$ This high event rate is alarming, especially because these patients had so far been considered to have a low risk, and may, as now confirmed by MADIT II, lead to a drastic change in implantation practice. ${ }^{6}$ The results show, furthermore, that serial testing with antiarrhythmic drugs does not seem to be necessary to prove that these patients are at risk. The importance of inducibility itself, which seems to be questionable after MADIT II, however, is not yet clarified. When comparing the non-inducible registry patients with randomised inducible patients in MUSTT (multicenter unsustained tachycardia trial), it becomes obvious that patients with non-inducible VT had a significantly better outcome than conventionally treated patients with inducible ventricular tachyarrhythmias. ${ }^{10-15}$ The two year mortality caused by arrhythmia in the registry patients who did not have inducible VT was $12 \%$ versus $24 \%$ in the nontreated patients with inducible VT. The difference was highly significant. This patient population is at least very similar with respect to the mean left ventricular ejection fraction of $29 \%$ versus $27 \%$ in our patient population. Besides, the percentage of patients previously treated with coronary bypass grafting was similar to that in our patients.

There are two differences between patients in the present study and the MADIT I population. Firstly, in the present series no epicardial ICD systems were implanted. A potential proarrhythmic influence of epicardial leads on the occurrence of VT/VF during follow up has always been a concern, especially early after implantation. However, there are no sound data that would allow a valid statement about the impact of the use of different lead systems. Secondly, it was argued that the ICD treatment arm in MADIT I may have had a better outcome because of a higher rate of patients taking $\beta$ blockers. However, in the present study, at last follow up $\beta$ blocking agents were taken by $69 \%$ of all patients and diuretics and angiotensin converting enzyme inhibitors by $73 \%$ and $83 \%$ of the patients, respectively. Yet the incidence of malignant ventricular tachyarrhythmias was high. This finding at least shows that, even under relevant heart failure medication, the risk of ventricular tachyarrhythmias is considerable. MADIT II showed that patients with a wide QRS had a higher risk of experiencing malignant ventricular tachyarrhythmias. ${ }^{6}$ Although the present population is rather small and the study was not randomised, our findings of a higher VT/VF event rate in patients with a QRS complex $>120 \mathrm{~ms}(68 \%)$ than in patients with a QRS width $<120 \mathrm{~ms}(36 \%)$ reflect the MADIT II results.

\section{Cycle length and morphology of tachyarrhythmias}

Data on the electrophysiological characteristics of arrhythmic events in patients with prophylactic ICD are lacking. In the present study the vast majority (92.3\% of all episodes) of recorded arrhythmic events were monomorphic tachycardias. Polymorphic VT and primary VF were rare, occurring in 7.7\% of all episodes. For the first time it is feasible to gain information about the nature of sudden death in patients from whom until now only recordings made by emergency personnel could be obtained, which most frequently documented VF. The findings of Rueppel and colleagues ${ }^{16}$ of recurrences in patients with aborted sudden death for VF showed that patients with a coronary artery disease had exclusively monomorphic VTs during later follow up. Moreover, the rate characteristics were comparable with those in the present study population with a mean cycle length of 282 ms. ${ }^{16}$ These observations are in accordance with early reports from Bayes de Luna and colleagues, ${ }^{17}$ who found that VT was the predominant underlying arrhythmia leading to ambulatory sudden death. At least for patients with coronary artery disease, prior myocardial infarction, and an ejection fraction below 35\%, we can now assume that sudden death is preceded by a monomorphic VT that degenerates into VF. This information is of value for the debate on downgrading (less expensive) ICD devices for primary prophylaxis that are lacking antitachycardia pacing options and provide only a limited number of shocks. In the present patient population antitachycardia pacing was substantially efficacious in 117 of 142 of the episodes. Use of these limited devices, therefore, should be obsolete. The high percentage of episodes with a cycle length below $320 \mathrm{~ms}$ (107 of 142 episodes) may be explained by the low rate of antiarrhythmic drug use by the present patients.

\section{Incidence of fast VT and potential clinical benefit of treatment}

One frequently used argument against implementation of the MADIT I or II criteria is that tachyarrhythmias treated by the device during follow up may not be life threatening. Furthermore, because of the short detection period of the devices for VT, detection may lead to overtreatment. However, the VT cycle length in the present study was shorter than $260 \mathrm{~ms}$ in $55.6 \%$ of the patients. It is very likely that VTs of more than 16 consecutive beats faster than 230 beats/min are "malignant" events. Böcker and colleagues ${ }^{18}$ have discussed the necessity of a surrogate end point of fast VT based on a cut off of 240 beats/min to assess the potential benefit of ICDs in their large patient series of ICD patients, who received an ICD for secondary prevention. ${ }^{18}$ When applying this surrogate end point of fast VT as a hypothetical death rate, the event-free rate after one year and 18 months in patients with coronary disease was $85 \%$ and $74 \%$, respectively. In the present study the rate of freedom from hypothetical death based on a cut off of a $260 \mathrm{~ms}$ cycle length was comparable, at $83.2 \%$ after one year and $78.4 \%$ after two years, with the one year and 18 month rates in their study. ${ }^{18}$ In a larger study by the same authors of 353 patients with coronary artery disease they found rates of freedom from hypothetical death of $78 \%$ after one year and $61.7 \%$ after three years. ${ }^{19}$

\section{Time interval between last $\mathrm{MI}$ and first VT/VF}

Finally, one striking result of the present study was the long interval between the last documented myocardial infarction and the first VT/VF event after ICD implantation. The widely used argument that a patient who has survived many years after myocardial infarction without syncope or documented VT has a low risk for a future event may therefore be challenged. Development of worsening heart failure in the course of coronary artery disease and postmyocardial infarction leading to increased left ventricular diameter, end diastolic pressure, and sympathetic activation may, among others, contribute to the described late risk of fatal ventricular tachyarrhythmias.

\section{Limitations}

This study was not a randomised trial. However, because of its prospective design this observational study confirms the outcome data of MADIT I. Mortality was not an end point; however, the high rate of potentially life threatening arrhythmic events (VF and VT with a $<260$ ms cycle length) may serve as a valuable surrogate end point.

\section{Conclusions}

Patients fulfilling modified MADIT I criteria should receive an ICD to prevent sudden death. There is a high incidence of VT and VF even a long time after a myocardial infarction. In the majority of patients the first arrhythmic event is 
monomorphic VT. Polymorphic VT and primary VF seem to be rare.

\section{Authors' affiliations}

C Wolpert, J Kuschyk, N Aramin, S Spehl, F Streitner, T Süselbeck, B Schumacher, K K Haase, R Schimpf, M Borggrefe, 1st Department of Medicine-Cardiology, University Hospital of Mannheim, Faculty of Clinical Medicine of the University of Heidelberg, Mannheim, Germany

\section{REFERENCES}

1 Moss AJ, Hall WJ, Cannom DS, et al. Improved survival with a defibrillator in patients with coronary disease at high risk for ventricular arrhythmia. N Engl J Med 1996;335:1933-40.

2 Anon. The Antiarrhythmics Versus Implantable Defibrillators (AVID) Investigators. A comparison of antiarrhythmic drug therapy with implantable defibrillators in patients resuscitated from near-fatal ventricular arrhythmias. N Engl J Med 1997;337:1576-83.

3 Connolly SJ, Gent M, Roberts RS, et al. Canadian implantable defibrillator study (CIDS): study design and organization. Am J Cardiol 1993;72:103F-8F.

4 Kim SG, Hallstrom A, Love JC, et al. Comparison of clinical characteristics and frequency of implantable defibrillator use between randomized patients in the antiarrhythmics vs implantable defibrillators (AVID) trial and nonrandomized registry patients. Am J Cardiol 1997:80:454-57.

5 Kuck KH, Cappato R, Siebels J, et al. Randomized comparison of antiarrhythmic drug therapy with implantable defibrillators in patients resuscitated from cardiac arrest: The cardiac arrest study Hamburg (CASH). Circulation 2000;102:748-54.

6 Moss AJ, Zareba W, Hall JW, et al. Prophylactic implantation of a defibrillator in patients with myocardial infarction and reduced ejection fraction. N Engl J Med 2002;346:877-83

7 Anon. ACC/AHA/NASPE 2002 guideline update for implantation of cardiac pacemakers and antiarrhythmia devices: summary article: a report of the
American College of Cardiology/American Heart Association task force on practice guidelines (ACC/AHA/NASPE committee to update the 1998 pacemaker guidelines). Circulation 2002;106:2145-61.

8 Hohnloser SH, Andresen D, Block M, et al. Guidelines for the implantation of implantable defibrillators. Z Kardiol 2000;89:126-234.

9 Priori SG, Aliot E, Blomstrom-Lundqvist C, et al. Update of the guidelines on sudden cardiac death of the European Society of Cardiology. Eur Heart $J$ 2003;24:13-5.

10 Buxton AE, Lee KL, DiCarlo L, et al. Electrophysiologic testing to identify patients with coronary artery disease who are at risk for sudden death. N Engl J Med 2000;342:1937-45.

11 Buxton AE, Hafley GE, Lehmann MH, et al. Prediction of sustained ventricular tachycardia inducible by programmed stimulation in patients with coronary artery disease: utility of clinical variables. Circulation 1999;99:1843-50.

12 Buxton AE, Marchlinski FE, Waxman HL, et al. Prognostic factors in nonsustained ventricular tachycardia. Am J Cardiol 1984;53:1275-9.

13 Buxton AE, Lee KL, Fisher JD, et al. A randomized study of the prevention of sudden death in patients with coronary artery disease. N Engl J Med 1999:341:1882-90

14 Mittal S, Lomnitz DJ, Mirchandani S, et al. Prognostic significance of nonsustained ventricular tachycardia after revascularization. J Cardiovasc Electrophysiol 2002;13:342-6.

15 Wilber DJ, Olshansky B, Moran JF, et al. Electrophysiological testing and nonsustained ventricular tachycardia: use and limitation in patients with coronary artery disease and impaired ventricular function. Circulation 1990;82:350-8.

16 Rueppel R, Schlueter CA, Boczor S, et al. Ventricular tachycardia during follow-up in patients resuscitated from ventricular fibrillation: experience from stored electrograms of implantable cardioverter-defibrillators. J Am Coll Cardiol 1998;32:1724-30.

17 Bayes de Luna A, Coumel P, Leclercq JF. Ambulatory sudden cardiac death: mechanisms of production of fatal arrhythmia on the basis of data from 157 cases. Am Heart J 1989;117:151-9.

18 Böcker D, Block $M$, Isbruch $F$, et al. Do patients with an implantable defibrillator live longer? J Am Coll Cardiol 1993;21:1638-44.

19 Böcker D, Bänsch D, Heinecke A, et al. Potential benefit from implantable cardioverter-defibrillator therapy in patients with and without heart failure. Circulation 1998;98:1636-43.

\section{ELECTRONIC PAGES}

\section{Heart Online case reports: www.heartinl.com}

7

he follow electronic only articles are published in conjunction with this issue of Heart.

\section{Incidental finding of a papillary fibroelastoma on the aortic valve in 16 slice multi-detector row computed tomography}

\section{A Bootsveld, J Puetz, E Grube}

Papillary fibroelastoma (PFE) is a benign, rare, gelatinous tumour derived from the endocardium, primarily the cardiac valves, which is usually diagnosed by high resolution echocardiography. Although rarely clinically symptomatic, PFEs have a potential for coronary ischaemia, systemic embolisation with neurologic symptoms, and sometimes valvar dysfunction. There are reports of coronary occlusion and even sudden cardiac death due to a ball valve phenomenon on the coronary ostia. This report describes the characteristics of a PFE with multidetector 16 slice computed tomography and 1.5 Tesla cardiac magnetic resonance imaging.

(Heart 2004;90:e35) www.heartjnl.com/cgi/content/full/90/ $6 / \mathrm{e} 35$

\section{Pseudo-pericardial tamponade after perforation of the right coronary artery}

C Steinwender, R Hofmann, F Leisch

A case of perforation of the right coronary artery, which was complicated by an intramural right ventricular haematoma with pseudo-pericardial tamponade resulting in fatal asystole, is presented.

(Heart 2004;90:e36) www.heartjnl.com/cgi/content/full/90/ $6 / \mathrm{e} 36$ 